\title{
Point de vue dialectique sur le modèle du traitement adaptatif de l'information et sur la thérapie EMDR
}

\author{
Brurit Laub \\ Nomi Weiner \\ Rehovot, Israël \\ Sheila S. Bender (1) \\ New Jersey, United States
}

\begin{abstract}
Cet article propose un point de vue dialectique sur le modèle du traitement adaptatif de l'information (TAI) de Shapiro $(1995,2001)$, avec une application à la thérapie EMDR (désensibilisation et retraitement par les mouvements oculaires). Les principes dialectiques peuvent contribuer à une compréhension plus détaillée de la manière dont le système TAI fonctionne et offrir de nouvelles lignes directrices thérapeutiques. Notre perspective dialectique est fondée sur deux propositions: La première dit que le mouvement du système TAI vers l'intégration se compose de deux mouvements dialectiques, l'un horizontal et l'autre vertical. Le mouvement horizontal a lieu entre divers opposés, comme le danger par rapport à la sécurité, la dépendance par rapport à l'indépendance, une mauvaise opinion de soi par rapport à une bonne opinion de soi. Le mouvement vertical, lui, concerne les mouvements tout/partie, dans lesquels un tout devient une partie du tout supérieur suivant. Le flux synergique de ces deux mouvements dialectiques est représenté sous la forme d'une spirale du système TAI. La deuxième proposition suggère que le système TAl fonctionne par cycles de différenciation et de liaison. Ces cycles découpent en morceaux le réseau mnésique condensé et fragmenté, ce qui permet l'apparition de nouveaux liens. Nous évoquons également les rapports de la différenciation et de la liaison avec l'accordage dialectique et avec l'attention double en pleine conscience. À l'aide de vignettes cliniques, nous montrons comment cette perspective peut fournir au thérapeute EMDR une carte du traitement associatif du patient, améliorer son accordage thérapeutique et favoriser des tissages cognitifs dialectiques efficaces lorsque le traitement se bloque.
\end{abstract}

Mots-clés : EMDR ; modèle du traitement adaptatif de l'information (TAI) ; dialectique ; liaison ; différenciation ; attention double en pleine conscience (ADPC)

$\mathbf{L}$ es nombreuses applications thérapeutiques de l'EMDR (désensibilisation et retraitement par les mouvements oculaires) s'appuient toutes sur le modèle du traitement adaptatif de l'information (TAI) qui en guide la pratique clinique (Shapiro, 1995, 2001, 2007). Ce modèle postule que pour donner du sens aux stimuli entrants, nous assimilons les nouvelles expériences dans nos réseaux mnésiques existants. Elles y sont traitées par "métabolisation" ou "digestion", ce qui donne lieu, pour la personne, à de nouveaux apprentissages et à une nouvelle guidance pour l'avenir (Shapiro, 2007). La pathologie apparaît lorsque des vécus traumatisants ou négatifs non traités sont stockés sous leur forme spécifique d'état,

This article originally appeared as Laub, B., Weiner, N., \& Bender, S. S. (2017). A dialectical perspective on the AIP model and EMDR therapy. Journal of EMDR Practice and Research, 11(2), 111-120. doi:10.1891/1933-3196.11.2.111. Translated by François Mousnier-Lompré, 2019. 
congelés dans le temps, dans leur propre réseau mnésique, et dans l'incapacité de se relier à d'autres réseaux mnésiques qui contiennent, eux, des informations adaptées. Au cours de la thérapie EMDR, les composantes de ces vécus stockés de façon implicite (les perceptions sensorielles, les émotions, les pensées, les sensations corporelles, les croyances) sont activées et progressivement reliées à des réseaux adaptés plus larges. 'L'expérience clinique a montré qu'une fois ces souvenirs spécifiques retraités, le sentiment de sa valeur et de son efficacité se modifie automatiquement chez le patient. Cela l'amène spontanément à des comportements nouveaux, plus positifs pour lui." (Shapiro, 2001).

Bergmann (2012) considère le modèle TAI comme une synthèse entre neurosciences et psychothérapie, avec à la fois le système physique de traitement de l'information, qui assimile les nouvelles expériences au sein des réseaux mnésiques déjà existants, et la mise en lien de réseaux neuronaux, mise en lien qui est en rapport avec l'expérience de la personne (pensées/croyances, images, émotions et sensations). De notre point de vue, la perspective dialectique permet de comprendre encore mieux cette synthèse.

\section{Les concepts dialectiques}

Les philosophies occidentales et orientales se penchent depuis des milliers d'années sur le concept de polarité et sur l'unité dynamique des opposés qui en résulte (Watts, 1963). Dans la philosophie classique grecque, la dialectique est une forme de dialogue, avec des propositions (thèse) et des contrepropositions (antithèse), qui produit une nouvelle synthèse (Williams, 1989). Dans la philosophie orientale, les opposés sont conçus comme les extrémités d'un tout unique, représenté, par exemple, par les énergies primordiales féminines et masculines du Yin et du Yang.

Laub and Weiner $(2007,2013)$ proposent une conceptualisation dialectique applicable à diverses approches psychothérapeutiques. Ils y incluent également une large analyse de l'importance des contraires et des synthèses qui en résultent, dans les formes anciennes (Harner, 1990) et modernes de la psychothérapie: thérapie jungienne (Jung, 1963), Gestalt (Perls, 1959), psychosynthèse (Assagioli, 1965), neuroprogrammation linguistique (Bandler \& Grinder, 1982), Voice Dialogue (Stone \& Winkelman, 1985), Somatic experiencing (Levine \& Frederick, 1997), thérapie comportementale dialectique (Lynch, Chapman, Rosenthal, Kuo, \& Linehan, 2006), cothérapie dialectique (Hoffman, Gafni, \& Laub, 1994; Hoffman
\& Laub, 2006) et thérapie dialectique fonctionnelle (Almagor, 2011).

\section{Une vue dialectique du modèle du traitement adaptatif de l'information}

Il existe déjà plusieurs éléments dialectiques dans la thérapie EMDR, même s'ils ne sont pas décrits comme tels: il y a les opposés cognition négative/cognition positive, les stimulations alternées à gauche et à droite (SBA), l'attention double portée à la fois au passé et au présent (observer le souvenir traumatique passé tout en restant présent dans la sécurité de la pièce de thérapie), et les associations adaptatives par opposition aux associations traumatiques. Les principes dialectiques ne sont pas explicitement élaborés dans le modèle TAI; nous avançons l'idée que leur élaboration améliorerait notre compréhension du fonctionnement du TAI et permettrait de faciliter ce dernier lorsque c'est nécessaire.

\section{Le mouvement spontané de traitement des informations vers une intégration plus élevée}

$\mathrm{Au}$ cœur du modèle TAI, il y a le postulat de l'autoguérison, de la tendance naturelle du système TAI à aller vers un état de bien-être (Shapiro, 2001). Ce point est en accord avec le postulat dialectique selon lequel le changement est fondé sur le mouvement spontané d'un processus développemental vers l'intégration optimale (Bopp \& Weeks, 1984). On peut se représenter cela comme un mouvement de traitement de l'information, s'élargissant vers une intégration plus élevée, dans laquelle la thématique négative devient plus adaptée et le self connaît une transformation positive.

\section{Le mouvement dialectique horizontal entre opposés}

Le modèle TAI est cohérent avec la compréhension qu'avaient les premiers théoriciens classiques Freud et Pavlov de ce qu'on appelle maintenant le traitement de l'information. "En particulier, il semble y avoir un équilibre neurologique dans un système physiologique distinct, qui permet au traitement des informations d'amener celles-ci à une 'résolution adaptée"' (Shapiro, 2001, p. 30). Dans le modèle TAI, le concept d'opposés est déjà présent chez Shapiro (2001): "La résolution, dit-elle, se produit par exemple quand les informations perturbantes, jusque-là isolées, sont mises en contact avec les informations adaptées déjà présentes chez le sujet” (pp. 31-32). 
Cette notion du partenariat d'opposés pour arriver à un équilibre est fort proche du postulat dialectique selon lequel le mouvement consiste en l'émergence successive d'une thèse, d'une antithèse et d'une synthèse (Bopp \& Weeks, 1984): nous considérons le passage d'associations inadaptées à des associations adaptées comme un mouvement dialectique horizontal entre des opposés particuliers de la personne: danger/sécurité, dépendance/indépendance. La synthèse résultante constitue une intégration plus élevée, avec un nouvel équilibre entre les contraires, menant à son tour à une autre synthèse, etc. Ce mouvement horizontal du système TAI peut être repéré dans le traitement EMDR et le thérapeute peut intervenir dialectiquement pour restaurer le système TAI.

\section{Cas clinique}

Une patiente EMDR traitait la perte récente de son bébé, une petite fille, lors d'une naissance prématurée. Vers la fin du traitement adaptatif, elle a imaginé un joyeux mariage pour ses jeunes fils, un jour futur, mais le bébé restait dans un coin. La patiente essayait, en vain, de se déconnecter de son chagrin. Le thérapeute lui a alors dit: "Vous avez un grand cœur, qui peut contenir à la fois le chagrin et le bonheur." Il avait choisi ce tissage cognitif pour suggérer à la patiente qu'elle pouvait contenir des émotions opposées et qu'elle n'avait pas besoin d'en supprimer une. Ce tissage dialectique a restauré le flux de son système TAI. À la série suivante de SBA, elle a dit:

Avant, je les voyais séparés [la peine et le bonheur], mais maintenant, j'arrive peu à peu à les relier, et le bébé n'est plus dans le coin. J'ai le droit d'être heureuse. Je peux revenir à l'hôpital [où elle avait accouché], alors que ça me faisait très peur. J'ai du chagrin ... mais je pourrai $y$ accoucher à nouveau.

\section{Le mouvement dialectique vertical des déplacements tout/partie}

Selon Shapiro (2001), le traitement EMDR produit une avancée rapide vers la santé ou vers des émotions positives, et vers une meilleure estime de soi. À notre avis, ces transformations peuvent s'expliquer par une autre hypothèse dialectique, selon laquelle l'univers fonctionne comme un organisme hautement différencié de systèmes interactifs, organisés hiérarchiquement au sein de systèmes plus larges (Bopp \& Weeks, 1984). Cette hiérarchie tout/partie est constituée d'ensembles qui deviennent les parties d'un ensemble supérieur (Koestler, 1978; Smuts, 1926, Wilber, 1996). Par exemple, les atomes forment des molécules, les molécules constituent des cellules, les cellules des organes, etc. Siegel (1999) a repris, de la théorie de la complexité mathématique, le principe de l'auto-organisation des systèmes; selon ce principe, les systèmes évoluent vers une complexité maximale ou vers un tout cohérent et harmonieux.

La séquence tout/partie des quatre niveaux de l'information. Wilber (1996) décrit une hiérarchie tout/partie avec quatre niveaux d'information: sensorimoteur, émotionnel, cognitif et spirituel. Dans un article antérieur, nous avons parlé de cette hiérarchie comme du mouvement dialectique vertical, qui se développe dans des déplacements tout/partie vers une intégration supérieure ou vers une complétude (Laub \& Weiner, 2013). Nous avançons l'idée que ce mouvement vertical peut expliquer la nature expansive du système TAI.

Le mouvement vertical permet au patient de passer d'une perspective partielle à une perspective plus complexe et plus complète: par exemple, pendant le traitement, un fragment sensorimoteur intrusif est intégré à une expérience sensorimotrice et émotionnelle plus complexe. Lorsque le système TAI s'élargit jusqu'au niveau cognitif, les trois niveaux d'information sont mieux intégrés et des prises de conscience apparaissent, avec une perspective plus élevée. Cela va de pair avec la transformation du thème négatif en un thème adapté. Parfois, le système TAI s'élargit encore jusqu'au niveau spirituel qui intègre les quatre niveaux d'information. Cette expansion s'accompagne de vécus transcendantaux de connexion à soi-même, aux autres et à l'univers.

La séquence tout/partie du fragment, de l'événement, de l'épisode, du thème et de l'identité. La séquence tout/partie du fragment, de l'événement, de l'épisode, du thème et de l'identité se repère plus facilement après un traumatisme récent (Laub \& Weiner, 2011; Shapiro \& Laub, 2008, 2009). Le protocole EMDR R-TEP (Recent Traumatic Episode Protocol), qui est une adaptation du protocole EMDR standard à des interventions précoces, définit l'épisode traumatique comme une série d'événements, depuis l'incident traumatique original jusqu'à aujourd'hui. L'épisode comprend des cibles multiples, comme des fragments intrusifs simples ou des expériences plus complexes, qui font partie des différents événements vécus au sein de l'épisode traumatique. Cette séquence a lieu en 
même temps que la séquence tout/partie des quatre niveaux d'information (sensorimoteur, émotionnel, cognitif, spirituel).

\section{Cas clinique}

Une patiente qui avait eu récemment un accident de voiture a été traitée avec l'EMDR R-TEP. Sa première cible était un fragment sensorimoteur intrusif simple: "le moment où la voiture est en l'air et où je vois des étincelles". Au cours du processus, elle s'est souvenue d'expériences plus complexes présentant des aspects sensorimoteurs et émotionnels, comme son appel téléphonique à son père, alors qu'elle se sentait anxieuse et perdue, et l'arrivée de celui-ci, qui a pris les choses en main. Le traitement de la patiente s'est développé à partir d'un "tout" sensorimoteur simple, qui est devenu une partie d'un tout plus grand avec plus de niveaux d'information. Au fil du traitement d'autres parties de l'épisode traumatique et de l'apparition de synthèses entre différents opposés, son thème cognitif d'hyper-responsabilité et de maitrise excessive a commencé à se transformer. La patiente s'est rendu compte qu'elle s'attribuait une trop grande responsabilité. Elle pouvait en abandonner une partie et rester tout de même responsable et maîtresse d'ellemême. Cette transformation du thème indiquait un nouvel équilibre entre maîtrise et manque de maîtrise, ce qui a produit une attitude plus souple envers ellemême et envers les autres, ainsi que des choix et des comportements nouveaux. Le retraitement, chez cette patiente, ne s'est pas élargi jusqu'au niveau spirituel.

La séquence tout/partie est décrite schématiquement pour illustrer l'expansivité naturelle du système TAI. Pendant la thérapie EMDR, les niveaux peuvent se chevaucher. Les patients ont leurs modèles de traitement personnels, qui commencent généralement par ce qui leur est le plus familier et passent plus tard à des niveaux moins directement accessibles. Si les changements tout/partie se bloquent, le thérapeute peut faire un tissage cognitif qui encourage le passage à un niveau d'information moins accessible (données sensorielles, sensations corporelles, émotions ou pensées). Cela renforce le mouvement dialectique vertical vers une intégration supérieure.

\section{La spirale du système de traitement adaptatif de l'information}

Nous avançons l'idée que le système TAI s'élargit vers une complexité, une harmonie et une intégrité plus grandes, grâce au flux synergique des deux mouvements dialectiques, représentés ici sous la forme d'une spirale (cf. Figure 1). Le système TAI oscille pendant le traitement des traumatismes, atteignant parfois un nouveau niveau d'intégration et redescendant parfois pour traiter d'autres expériences implicites de vulnérabilité. Au début du traitement des traumatismes, la spirale du système TAI est large, parce que les opposés de la personne sont éloignés, sans connexion et avec peu de mouvements dialectiques entre eux; la perception de l'individu est alors rigide et unidimensionnelle. Elle est également partielle, parce que le mouvement dialectique vertical est limité et les parties ne se transforment pas en ensembles plus grands. Pendant le traitement, le mouvement dialectique horizontal commence à se faire naturellement et les contraires se rapprochent l'un de l'autre, produisant graduellement de nouvelles synthèses. Au fur et à mesure que le traitement s'élargit, des mouvements verticaux apparaissent.

C’est ce qui est représenté par le rétrécissement du mouvement en spirale vers le haut, lorsque la personne acquiert une perspective plus équilibrée et plus complète. En comprenant la nature oscillante du système TAI, le thérapeute peut améliorer ses choix cliniques quant au moment et à la manière dont il doit intervenir si le retraitement est bloqué.

\section{Cas clinique}

L'exemple suivant illustre la nature oscillante du système TAI. Il présente également l'utilisation de tissages dialectiques qui rendent les opposés plus accessibles, facilitant le mouvement dialectique horizontal et la validation des mouvements verticaux par le thérapeute. La patiente était en thérapie depuis quelques années et elle y avait traité des abus sexuels, subis dans son enfance, qui mettaient à mal sa relation conjugale. Elle a demandé une séance d'EMDR pour traiter une situation future qui l'inquiétait beaucoup: elle devait chanter sur scène ses propres chansons. Dès le début, le traitement associatif a fluctué, se déplaçant horizontalement entre différents opposés, comme fuir la scène ou y rester, éviter les spectateurs ou leur faire plaisir, se sentir incompétente ou compétente. Ensuite, une synthèse a émergé quand elle s'est rappelé une chanson qu'elle avait écrite, intitulée "Je suis super-bien là où je suis". Lors de la série suivante de SBA, la patiente dit:

C'est lié au traumatisme [mouvement dialectique vertical descendant], par rapport à la façon dont je me suis comportée dans ces moments [des abus]. Je ne sais pas ce qui se passe ... et 
je suis... totalement dans un endroit différent et je me sens merveilleusement bien [dissociation]. Mais ce n'était pas merveilleux du tout; je me sentais très malade, je souffrais beaucoup. Encore une fois, c'est deux pôles, vous le voyez?

Le thérapeute lui répond: "Bon. Remarquez ce qui se passe lorsque vous allez de l'un à l'autre ..." (Le thérapeute encourage la patiente à remarquer les contraires afin de faciliter le mouvement dialectique horizontal.) Patiente: "J'ai besoin d'un mouchoir, OK; je vais le remarquer, mais maintenant c'est embêtant: c'est là. Cette saloperie de trauma est partout." Thérapeute: "Laissez venir ce qui vous vient, laissez simplement passer et notez ce qui vous vient, comme dans un film." (Le thérapeute est attentif à la nécessité d'introduire de la distance par opposition au sentiment d'accablement de la patiente, en facilitant le mouvement dialectique.) La patiente dit:

Je crois que c'est comme la méthode Coué: j'entends cette chanson, "Je me sens super-bien là où je suis" et ça continue: "Je m'aime comme je suis". Je la chante, mais la seule chose que j'aie envie de faire, c'est de crier: je ne suis pas bien du tout, je ne me sens pas super-bien. Je n'ai pas de conjoint, j'ai eu ce traumatisme de merde et c'est difficile, et je veux avoir des enfants et une famille, et ce n'est pas super-bien du tout d'avoir été violée.

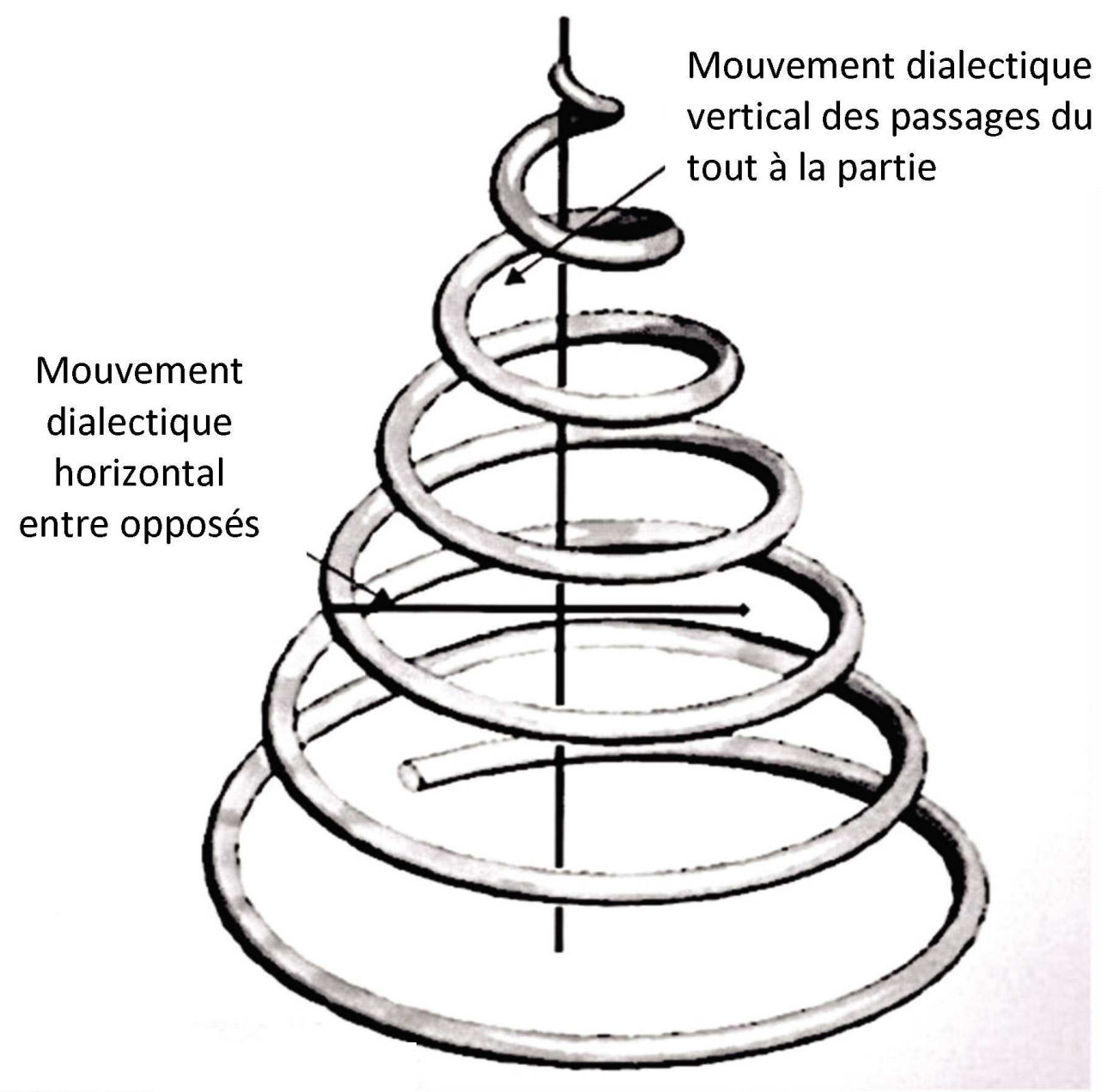

FIGURE 1. La spirale du système de traitement adaptatif de l'information. 
Le thérapeute demande doucement à la patiente de rester dans cette émotion pendant quelques minutes (il l'encourage à être avec sa colère parce que cet état était dialectiquement opposé à son habituel déni.) Après la série suivante de SBA, la patiente dit: "Et alors, tout me semble vide et fade." Thérapeute: "Oui, où ressentez-vous ça dans votre corps?" (Il tâche d'aider sa patiente à se relier à ses sensations corporelles et de les lui rendre plus accessibles pour le traitement, au lieu de s'en trouver dissociée. Cela peut améliorer le mouvement dialectique horizontal.) Patiente: "En moi, dans ma tête, ma gorge, ma poitrine, comme ça ...” Après une série de SBA, elle dit:

Il y a plein de choses que je connais. Je commence à entendre ces endroits qui m'ont fait penser que je devais chanter ce genre de choses, par exemple la poésie amérindienne. C'est comme un continuum de temps, des années, une sorte de croissance par laquelle je passe, et son origine est quelque part. (Cette ressource indique un déplacement vertical vers un niveau spirituel - elle s'approche du ressenti de son propre centre, tout en passant à une perspective plus large).

\section{Elle poursuit:}

Je me sens vide, debout, sur la scène, et puis ... Ça commence à jouer dans ma tête. J'ai un peu l'impression d'entendre vraiment l'eau qui babille. [L'eau qui babille était une ressource antérieure, un sentiment d'être intérieurement en harmonie tout en étant avec des amis, en correspondance dialectique avec ses peurs.] Ça me donne de la perspective, ça me donne beaucoup plus d'espace pour voir le moment où je monte sur la scène.

Le thérapeute lui dit: "C'est très important, ça. Estce que cela ouvre quelque chose pour vous?" (Il valide ce mouvement vertical, en suggérant qu'il pourrait se développer davantage encore.)

Après une autre séquence de SBA, la patiente dit:

"Ça m'ouvre à plein de choses, j'entends les gens dans le désert, et avant ça, ces musiques, ou ces danses, qui viennent de quelque part. Ça m’apporte un message de ... c'est bien ... immerge-toi dans cette expérience [sur la scène]. Si l'expérience n'était pas bonne pour tout le monde, elle n'aurait pas eu lieu ... C'est de là que je viens vraiment ... Je sais très bien pourquoi je fais ça. (Une fois que la patiente a pu entrer en contact avec sa douleur, sa solitude et sa colère, elle se déplace horizontalement vers un lieu de confort, de lien et d'harmonie. Retrouvant sa valeur personnelle, elle fait un changement vertical avec une perspective plus large sur elle-même).

À la fin de la séance, elle ressent une profonde tranquillité et dit: "Je vois que je vais gérer tout ce qui va se présenter ce soir-là; c'est ma voie, c'est ce que je veux faire, je veux passer par là. Je sais que je vais assurer." Le thérapeute lui demande: "Quelles sont les sensations corporelles qui vont avec cet endroit?" (Le thérapeute essaye de lui rendre son centre plus accessible pour en faciliter l'intégration à tous les niveaux d'information.)

Oui, c'est bizarre. Dans mon corps, $\mathrm{j}$ 'ai un peu l'impression d'aller sur scène avec les talons que j'ai mis ce soir, ceux que je prends quand je fais des percussions. C'est une sorte de position debout ... Et j'ai l'impression de porter le costume de scène ... Je prends cette occasion, je prends cette excitation, je sais ce qu'il faut à mon corps parce que je l'écoute depuis un moment, et je le canalise sur quelque chose qui va m'aider à me tenir debout." (Se tenir debout entre ciel et terre émerge ici comme l'impression que son centre se transforme en un nouveau sentiment de totalité. Son système TAI s'élargit au niveau spirituel, où se fait une transformation du soi [de son identité].)

\section{Différenciation et liaison sont des aspects complémentaires du système de traitement adaptatif de l'information}

La différenciation et la liaison sont des notions dialectiques supplémentaires, qui peuvent enrichir notre compréhension du système TAI. La liaison se rapporte à la tendance à faire partie, à se connecter, à se rapprocher, à s'identifier à, et à appartenir à. La différenciation concerne au contraire la tendance à être à part, à séparer, à mettre à distance, à mettre une frontière, et à être autonome. Ce sont des processus développementaux de base, nommés différemment par divers auteurs: séparation et connexion (Klein, 1976; Laub \& Weiner, 2007; Pipp, 1990), autonomie et intimité (Bowlby, 1973), individuation et fusion (Bowen, 1978), autodéfinition et relation interpersonnelle (Blatt, 1995), et action et communion (Wilber, 1996). Siegel (2010) définit l'intégration comme la liaison de parties différenciées d'un système. 


\section{L'interaction dialectique liaison/différenciation}

Dans le modèle TAI, on met l'accent sur la liaison, qui établit des liens, au cours du traitement EMDR, entre les expériences traumatiques activées et des réseaux mnésiques adaptés plus larges (Shapiro, 2001). Nous avançons, quant à nous, que la différenciation est un processus tout aussi important, et complémentaire de la liaison. Cette interaction dialectique est cruciale pour le fonctionnement du système TAI: la différenciation, le processus qui sépare les expériences condensées stockées de façon dysfonctionnelle en différentes parties, et la liaison, le processus qui les relie à nouveau.

Nous suggérons en outre que le système TAI opère à travers des cycles de différenciation et de liaison. Le réseau mémoriel traumatique, condensé et fragmenté, est différencié en un nombre croissant de données sensorielles, de sensations corporelles, d'émotions, de pensées ou de diverses combinaisons de celles-ci, de sorte qu'elles puissent se relier de façon nouvelle. Nous avançons également que, par nature, ces cycles se déplacent entre des contraires, ce qui permet des synthèses nouvelles et une expansion vers une intégration supérieure.

\section{Déséquilibre entre différenciation et liaison}

La différenciation et la liaison s'appliquent également aux effets perturbateurs du trauma. Le trauma peut se manifester par des schémas diphasiques de symptômes psychoformes et somatoformes (Chu, 1998; van der Hart, van Dijke, van Son, \& Steele, 2000): d'une part, on a des symptômes comme l'engourdissement, l'hypoactivation et l'évitement; et d'autre part, des symptômes de flash-backs, d'hyperactivation et de perte de contrôle de soi. Le premier groupe indique trop de différenciation, le second un liage excessif. Le traitement adaptatif des traumatismes restaure l'équilibre entre différenciation et liaison. Si le thérapeute comprend où se situe son patient sur ce continuum, il peut utiliser des interventions dialectiques pour rétablir l'équilibre.

\section{Cas clinique}

L'exemple suivant montre l'expansion des cycles de différenciation et de liaison pendant le traitement adaptatif. La patiente a entrepris une thérapie courte et ciblée, deux mois après l'inondation de sa maison. Elle a été traitée avec le protocole EMDR R-TEP (Shapiro \& Laub, 2008, 2009, 2013). La première cible était un fragment d'une sensation intense d'inconfort d'avoir dû passer deux jours dans l'appartement en mauvais état de sa voisine. Lors de la première séquence de SBA, ses associations étaient surtout sensorimotrices, différenciant son inconfort en différentes parties, comme sa difficulté à prendre une douche dans une salle de bain sale ou à prendre un repas dans une cuisine en désordre (cette femme pointilleuse était submergée par le dégoût, avec une liaison excessive à cette émotion). Après la série suivante de SBA, elle a dit: 'Là, je me suis dit qu'elle [la voisine] avait été très perturbée par l'inondation et qu'elle n'avait pas changé le linge de table" (la patiente a pu se distancier un peu et créer un lien adaptatif, permettant ainsi un mouvement dialectique horizontal entre la critique et l'acceptation). Elle a continué à différencier et à lier d'autres parties de l'épisode: "Les autres pièces étaient rangées; la salle de bain et la cuisine seules étaient mal rangées, mais pas sales." Plus tard, elle a traité ses sentiments d'être en "dette de gratitude" envers la voisine et a exprimé le désir de s'en libérer (elle a différencié son désir de liberté de son sentiment d'obligation, facilitant un mouvement dialectique horizontal.) Au fur et à mesure que le processus avançait, elle devenait plus souple et acceptait mieux la voisine et elle-même, en se reliant à un sentiment authentique de gratitude: "Je peux remercier ma voisine sans avoir l'impression de devoir la rembourser." Le traitement de cette patiente impliquait une différenciation de diverses parties et de divers aspects de son souvenir traumatique, permettant l'établissement de nouveaux liens. Au fil de l'avancée de son traitement adaptatif, des cycles de différenciation et de liaison se sont élargis en une intégration plus élevée, transformant sa crainte première de se sentir obligée en un sentiment de liberté et de gratitude authentique.

\section{Une vision dialectique de la relation thérapeutique}

Shapiro (2001) souligne l'importance d'établir une alliance thérapeutique solide dès le début du traitement EMDR, car les patients, pouvant être extrêmement vulnérables, ont besoin que le thérapeute leur fasse passer un message de sécurité, de souplesse et de préoccupation inconditionnelle envers eux.

\section{L'accordage dialectique}

Nous soulignons l'importance de l'accordage dialectique dans la relation thérapeutique: on entre délicatement en résonance avec les blessures d'attachement sous-jacentes du patient (liage), mais on invite aussi celui-ci à se développer (différenciation). Le 
thérapeute s'aperçoit de l'existence de parties niées ou non développées chez le patient, et il les amène au premier plan, d'une manière empathique et accordée. Cela favorise un mouvement dialectique horizontal chez le patient entre des opposés comme la peur et le courage, la faiblesse et la force.

Le thérapeute crée également une "expérience réparatrice", en répondant au patient de manière dialectiquement opposée à ce qu'il a connu de négatif dans ses relations avec les personnes importantes de son entourage. Cela peut renforcer la dyade thérapeutique et créer de nouveaux réseaux mnésiques adaptés. Ces aspects dialectiques correspondent à la conceptualisation avancée par Dworkin and Errebo (2010) de l'EMDR comme une thérapie à deux personnes qui recourt à un dialogue entre clinicien et patient, portant sur la résonance mutuelle, l'accordage émotionnel entre eux et la nature de leur relation.

\section{Cas clinique}

L'exemple suivant illustre le rôle de l'accordage et des tissages dialectiques pour favoriser le mouvement dialectique, en cas de blocage du retraitement. Ce patient était venu en thérapie en se plaignant de crises anxieuses quotidiennes, ainsi que de douleurs dans le côté inférieur droit du corps. C'était sa première séance d'EMDR. Cet homme était beaucoup dans le contrôle de soi et l'autocritique, ayant grandi avec des parents qui avaient du mal à admettre les sentiments de peur et d'impuissance. L'événement traumatique était une attaque de panique vécue à l'âge de 10 ans. Il avait réveillé sa mère, qui avait tâché de le calmer et lui avait demandé ce qui s'était passé, mais il n'avait pas pu traduire en mots ce qu'il ressentait. Après avoir un peu plus différencié l'événement, il a senti une légère pression dans sa poitrine. La thérapeute lui a dit: "OK, bon, votre corps commence à vous parler maintenant, soyez attentif, observez simplement ..." (dialectiquement accordée à la difficulté du patient à exprimer en mots son expérience, elle mettait en relief son langage corporel.) Après une séquence de SBA, le patient a dit: "Cela me conduit maintenant ... aux crises d'angoisse que j'ai maintenant." Après une autre séquence de SBA, il a dit: "Je suis un peu bloqué." Thérapeute: "Pas de souci; rien ne vient?" Patient: "Non." Thérapeute: "Alors, pistez simplement votre corps ... vous pouvez le faire sans effort" (la thérapeute parlait d'un ton apaisant, en normalisant l'inconfort du patient qui se sentait bloqué et en lui suggérant une observation de pleine conscience, sans effort). Après une autre séquence de SBA, le patient a dit: "Oui, il y a une sensation de pression autour de mes poumons, de mon ventre, un peu sur le côté droit, comme d'habitude ..." Thérapeute: "OK, très bien, maintenant ça apparaît, cet endroit ... remarquez simplement ce qui vous vient." (La thérapeute se montrait encourageante et intéressée.) Après une autre séquence de SBA, le patient a dit: “C'est tout, j'ai juste senti que j'avais besoin de respirer profondément pendant un moment, de prendre de l'oxygène." Thérapeute: "Oui ... est-ce que vous le faites en ce moment?" (Elle accompagnait son patient, en accordage étroit avec son "enfant traumatisé", effrayé et tout seul). Après une autre séquence de SBA, le patient a dit: "Je l'ai fait avant. J'essaie de me rappeler ce qu'il y avait d'autre, quand je me suis réveillé terrifié au milieu de la nuit et que j’appelais ma mère; mais je ne trouve rien. Peut-être est-ce trop loin pour que je me souvienne de ce qu'il y a eu." Thérapeute: "Oui, mais votre corps se souvient, lui; revenez à votre corps ..." (L'encouragement de la thérapeute était dialectiquement accordé au sentiment d'échec $\mathrm{du}$ patient, pour l'encourager à faire un mouvement dialectique horizontal entre des sentiments d'incompétence et de compétence). Après une autre séquence de SBA, le patient a dit: "En repensant à mes dix ans, c'était un sentiment de perte totale de contrôle, c'est ça qui m'est arrivé ... mais pourquoi ça ne s'en va pas? Pourquoi ça dure si longtemps? Quelquefois, c'étaient vraiment des heures et des heures de souffrance." Après une autre séquence de SBA, il a dit: "J'ai toujours eu peur que mes parents ne me croient pas si je leur disais qu'il m'arrivait quelque chose ... Je ne sais pas pourquoi. Ils ne m'ont pourtant jamais donné l'impression que je ne pouvais pas leur parler. Ils ne m'ont jamais dit que c'était mal de ressentir des choses comme ça. Je suis simplement arrivé très jeune à cette conclusion. Comme si quelque chose m'arrivait maintenant et que je le garde pour moi." (La thérapeute était accordée aux craintes de son patient, elle les reconnaissait et les validait, par opposition à ce qu'il avait vécu avec ses parents. Elle était sans cesse avec lui, dans les petites nuances des sensations de son corps, afin qu'il commence à sentir que tout ce qu'il vivait dans son corps était OK.) Thérapeute: "Bon, alors soyez attentif à ce besoin de le garder pour vous-même." (Elle tentait de l'aider à remarquer les sentiments qu'il rejetait, pour favoriser un mouvement dialectique horizontal entre leur dissimulation et leur partage). Patient: "C'est très lourd; je cache un cadavre dans le placard ... Je suis de nouveau bloqué." Thérapeute: "OK, on va vérifier maintenant; vous savez que de temps en temps, on fait ce contrôle. Si nous revenons au souvenir cible, notez si quelque chose a changé?" Patient: "C’est comme si 
je ne pouvais pas pointer du doigt: je crois que c'est vraiment la question qui m'a rendu dingue pendant toutes ces années. Qu'est-ce qui a bien pu aller de travers, bon sang? Ah ... attendez ... J'essaie de me rappeler: quand j'avais 10 ou 12 ans, j'ai eu l'appendicite" (il se reliait maintenant à l'expérience d'une appendicectomie à l'âge de 10 ans, ce qui expliquait ses douleurs dans le côté droit pendant les attaques de panique.) La thérapeute s'est alors montrée dialectiquement accordée, de façon verbale et non verbale, avec "l'enfant traumatisé", dans sa solitude, sa peur et son impuissance; elle lui a offert une expérience réparatrice dans laquelle, contrairement à ses parents, elle n'avait pas peur de l'expression de sa faiblesse. Le patient a pu dès lors se sentir plus en sécurité en se liant à ces vécus effrayants, et il a pu les différencier nettement. La thérapeute est restée proche, active et contenante, pour lui faire sentir qu'elle était présente avec lui, ce qu'il n'avait jamais connu en grandissant. Elle a encouragé l'expression des émotions désavouées par le patient pour en faciliter l'intégration, en l'aidant à accepter à la fois sa vulnérabilité et sa force.

\section{Une vision dialectique de l'attention double}

Pour F. Shapiro (1995, 2001), l'attention double est un élément essentiel de la thérapie EMDR. L’attention double met en lien la conscience simultanée des souvenirs traumatiques passés et de la sécurité actuelle du cadre thérapeutique. Shapiro (2001) souligne à la fois le rapprochement et la mise à distance quand elle écrit:

Il se peut que l'efficacité de l'EMDR vienne de sa capacité à produire exactement le juste équilibre entre la reviviscence de perturbations émotionnelles et l'obtention d'une position "d'observateur" sans jugement par rapport à l'émotion et au flot des associations somatiques, affectives, cognitives et sensorielles qui surgissent ...

Nous suggérons que la capacité d'attention double implique le mouvement dialectique du patient qui passe entre l'interne et l'externe, le passé et le présent, le danger et la sécurité - et qui se relie au souvenir traumatique et s'en différencie. Il y a également l'élément dialectique d'une alternance permanente entre le centrage intrapersonnel sur les souvenirs traumatiques et le partage interpersonnel avec un thérapeute témoin et émotionnellement accordé.

\section{La pleine conscience}

Dans les exercices de pleine conscience, on encourage les gens à être conscients de leurs sensations, de leurs sentiments et de leurs pensées, sans les juger (Kabat-Zinn, 1990; Siegel, 2007). En thérapie EMDR, la capacité de rester présent tout en traitant des expériences difficiles est renforcée par des instructions de pleine conscience, comme "simplement remarquer", "laisser venir ce qui vient" (Solomon $\&$ Shapiro, 2008). Siegel (2007) considère la pleine conscience comme la capacité d'empathie du soi observateur envers le soi qui vit l'expérience. Il évoque quatre aspects de l'attention: la curiosité, l'ouverture, l'acceptation et l'amour (COAA).

\section{L'attention double en pleine conscience}

Une séquence tout/partie d'attention double en pleine conscience. Inspirés par la notion développée par Siegel (2007) de quatre composantes dans la pleine conscience, nous avons remarqué que, pendant le traitement adaptatif, l'attention double se développe par mouvements tout/partie, et passe de l'ouverture à la curiosité, puis à l'acceptation des contraires et à l'amour. Nous parlons d'attention double en pleine conscience (ADPC) pour décrire la tendance naturelle à l'élargissement qu'on voit dans l'attention double. Cette séquence tout/partie de l'ADPC va de pair avec l'expansion des niveaux d'information, et on peut la repérer dans le traitement du patient:

- Ouverture. Le patient commence à ressentir un sentiment de sécurité qui améliore sa capacité d'observer son expérience traumatique. S'il se sent en grand danger, l'ADPC est compromise (submergée et/ou déconnectée) et le traitement ne peut pas continuer. Cet arrêt est une réaction sensorimotrice au danger. Le patient peut reprendre le traitement et retrouver sa capacité d'ADPC lorsqu'il retrouve un sentiment de sécurité.

- Curiosité. Une fois établi un minimum de sécurité, le système TAI peut s'élargir et passer au plan émotionnel: on voit alors apparaître des liens nouveaux et surprenants. Le patient s'implique avec curiosité dans le traitement associatif qui se déroule. L'ADPC est maintenant ouverte et curieuse.

- Acceptation des opposés. Le patient commence à faire confiance au processus et à la relation thérapeutique, et il a davantage d'ouverture et 
de curiosité: il ose contacter les points de vulnérabilité et les aspects contradictoires de son propre self. Cela renforce un mouvement dialectique horizontal vers des ressources, qui s'opposent aux points de fragilité. À mesure que la synthèse et l'acceptation de ces opposés augmentent, de nouvelles prises de conscience apparaissent. À cette étape, le système TAI s'élargit pour passer au niveau cognitif et le thème commence à se transformer. L'ADPC est maintenant ouverte, curieuse et accueillante.

- Amour. Au fur et à mesure que le système TAI s'élargit et passe au niveau spirituel, la compassion et l'amour du patient envers lui-même, les autres et l'univers se développent, permettant une transformation de soi. Elle s'accompagne du sentiment puissant d'être centré et entier. L'ADPC est maintenant ouverte, curieuse, accueillante et aimante.

L'interaction entre accordage dialectique et attention double en pleine conscience (ADPC) dans le système du traitement adaptatif de l'information.

L'accordage dialectique et l'ADPC interagissent pendant la thérapie EMDR et leur collaboration accélère l'intégration.

\section{Cas clinique}

L'exemple suivant illustre l'interaction dynamique entre l'accordage émotionnel dialectique et l'ADPC dans le système TAI. Une patiente, venue à la suite d'un divorce difficile, rapporte un traumatisme: elle a entendu un échange humiliant entre son ex-beaupère et son ex-mari. Juste au début du retraitement, elle demande qu'on arrête, parce qu'elle est submergée par ses émotions (la capacité d'ADPC de la patiente se ferme, ce qui bloque le traitement du système TAI). La thérapeute l'encourage doucement à rester en contact avec sa détresse: "juste encore un petit peu” (la thérapeute aide à ouvrir l'ADPC de la patiente en s'accordant dialectiquement à son manque de confiance dans sa capacité à contenir sa détresse. Elle exprime sa conviction que la patiente peut le faire.) La patiente est d'accord pour continuer à traiter et ressent rapidement un soulagement (l'ADPC de la patiente s'est ouverte, pendant que son système TAI se déplace dialectiquement entre détresse et soulagement.) Elle dit: "Je peux respirer; je me sens soulagée; je peux me voir de l'extérieur" (l'ADPC de la patiente est passé d'une liaison excessive à une certaine différenciation par rapport à l'expérience traumatique). Après la séquence suivante de SBA, elle déclare: “... ce n'est pas moi” (l'objet de cette attaque humiliante). Au fur et à mesure de son traitement, elle se rappelle que son père, lui aussi, était dévalorisé par son ex-beau-père. Maintenant, elle regarde le comportement de son père avec curiosité et empathie. Elle peut considérer différemment le fait qu'il ait accepté les humiliations que lui faisait subir son ex-beau-père. Ce n'était pas un acte de lâcheté, mais un noble acte d'amour qui l'a aidée à obtenir un divorce rapide (au fil de l'élargissement du traitement, elle accepte sa propre humiliation et celle de son père en se sentant plus digne. Son ADPC s'étend jusqu'à pouvoir accepter des contraires). Ensuite, son système TAI passe au traitement de sa colère contre son ex-beaupère. La thérapeute est en empathie avec son besoin de décharger sa colère. À la fin de la séance, la patiente peut avoir une certaine compassion envers lui, comprenant que son comportement reflète à la fois ses propres insécurités et son désir d'aider son fils (l'ADPC de la patiente s'est approchée d'un niveau spirituel de douceur aimante.)

\section{Discussion}

Une vision dialectique de l'action transformatrice de la thérapie EMDR

F. Shapiro (2001) décrit l'action transformatrice du traitement EMDR comme une "digestion" ou une "métabolisation". Nous suggérons que les principes dialectiques peuvent contribuer à la compréhension de ce processus de "digestion". Nous avançons l'idée que le système TAI procède par cycles de différenciation et de liaison. Pendant le traitement EMDR, le réseau mnésique activé, condensé, fragmenté et stocké de façon dysfonctionnelle, se trouve différencié en diverses parties et de nouveaux liens apparaissent, ce qui permet d'autres différenciations et d'autres liaisons. Ces cycles de différenciation et de liaison tendent à se déplacer horizontalement entre différents opposés au sein de l'individu, créant ainsi de nouvelles différenciations et de nouveaux liens entre le réseau mnésique traumatique et les réseaux mnésiques adaptatifs. Ils ont également tendance à se développer verticalement, dans des changements tout/partie, vers une intégration plus élevée. Le flux synergique des mouvements dialectiques horizontaux et verticaux crée le mouvement oscillant et en spirale du système TAI. 
La différenciation et la liaison, composantes indispensables de l'intégration

La liaison associative est un élément essentiel du modèle TAI, qui met moins en relief le rôle de la différenciation. Celle-ci est impliquée dans la notion de distanciation psychologique, c'est-à-dire à la capacité de considérer les expériences traumatiques d'un point de vue détaché (Gunter \& Bodner, 2009; Lee, Taylor, \& Drummond, 2006; Maxfield, Melnyk, \& Hayman, 2008). Ce point est similaire à ce qui a été suggéré ici concernant l'ADPC. Bien que nous suggérions ici une application plus large de cette notion, il semble que la distanciation psychologique pointe vers un seul aspect de la différenciation. Notre présente proposition considère à la fois la différenciation et la liaison comme des éléments indispensables du processus d'intégration. Ils sont également cruciaux dans l'ADPC et dans l'accordage émotionnel dialectique.

\section{Appliquer la perspective dialectique en thérapie EMDR}

Une carte $d u$ traitement associatif en expansion et des lignes directrices pour faire des tissages dialectiques. Appliqués au système TAI, les principes dialectiques fournissent au thérapeute EMDR une carte du traitement en expansion chez le patient. Ils lui offrent aussi des directives pour construire des tissages dialectiques qui faciliteront de diverses façons les mouvements dialectiques verticaux et horizontaux du système TAI: le thérapeute favorisera le mouvement horizontal en rendant accessibles les opposés et en validant les ressources du patient; et il facilitera le mouvement vertical tout/partie en encourageant l'intégration des niveaux d'information (sensorimoteur, émotionnel, cognitif et spirituel) et en validant les mouvements verticaux.

Une carte de l'attention double en pleine conscience (ADPC) en expansion et des lignes directrices pour construire des tissages dialectiques. Les tissages dialectiques peuvent également équilibrer l'ADPC, améliorant ainsi son expansion, de l'ouverture à la curiosité, puis à l'acceptation des contraires et enfin à l'amour. Cela va de pair avec l'expansion des quatre niveaux d'information, qui traduit une perspective plus large et plus complète chez le patient. Le fait de comprendre la nature en expansion de l'attention double peut aider le thérapeute à s'accorder émotionnellement à l'ADPC du patient, qu'elle soit bloquée ou au contraire en expansion, et à intervenir dialectiquement si c'est nécessaire.
L'accordage dialectique et les directives pour la construction de tissages dialectiques. Les tissages dialectiques sont également une part indispensable de l'accordage dialectique. Le thérapeute résonne, de façon verbale et non verbale, avec les blessures du patient et, quand c'est nécessaire, recourt à des tissages dialectiquement opposés à l'expérience traumatisante de celui-ci. L'accordage dialectique favorise l'oscillation du système TAI vers des endroits plus implicites et plus fragiles, et donc vers une guérison plus complète.

Des tissages dialectiques favorisant le mouvement en spirale $d u$ système de traitement adaptatif de l'information. Les tissages dialectiques favorisent le mouvement en spirale du système TAI: mouvement descendant vers des niveaux moins accessibles qu'il faut traiter, ou mouvement ascendant vers des niveaux supérieurs d'intégration et de complétude. Certains des tissages cognitifs dialectiques suggérés dans cet article sont utilisés en thérapie EMDR. Nous pensons cependant que l'adoption d'une perspective dialectique offre au thérapeute EMDR un cadre conceptuel qui lui permet de créer des interventions très fines. Elle favorise de nouvelles façons créatives d'être présent, de faire des tissages précis et de produire de nouvelles procédures. Un exemple de procédure nouvelle est l'enveloppe de connexion aux ressources, dans laquelle le patient accède à des ressources uniques à chaque séance EMDR (Laub, 2001).

\section{Bibliographie}

Almagor, M. (2011). The functional dialectic system approach to therapy for individuals, couples, and families. Minneapolis, MN: University of Minnesota Press.

Assagioli, R. (1965). Psychosynthesis: A collection of basic writings. London, England: Hobbs, Dorman: \& Company.

Bandler, R., \& Grinder, J. (1982). Reframing: Neuro-linguistic programming and the transformation of meaning. Moab, UT: Real People Press.

Bergmann, U. (2012). Neurobiological foundations for EMDR practice. New York, NY: Springer Publishing.

Blatt, S. J. (1995). The destructiveness of perfectionism: Implications for the treatment of depression. American Psychologist, 50, 1003-1020. doi:10.1037/0003066X.50.12.1003

Bopp, M. J., \& Weeks, G. R. (1984). Dialectical metatheory in family therapy. Family Process, 23, 49-61. doi:10.1111/j.1545-5300.1984.00049.x

Bowen, M. (1978). Family therapy in clinical practice. Northvale, NJ: Jason Aronson.

Bowlby, J. (1973). Attachment and loss: Vol. 2. Separation: Anxiety and anger. Middlesex: Penguin Books. 
Chu, J. (1998). Rebuilding shattered lives: The responsible treatment of complex post-traumatic and dissociative disorders. New York, NY: Wiley.

Dworkin, M., \& Errebo, N. (2010). Rupture and repair in the EMDR patiente/clinician relationship: Now moments and moments of meeting. Journal of EMDR Practice and Research, 4(3), 113-123. doi:10.1891/1933-3196.4.3.113

Gunter, R. W., \& Bodner, E. (2009). EMDR works... but how? Recent progress in the search for treatment mechanisms. Journal of EMDR Practice and Research, 3(3), 161-168. doi:10.1891/1933-3196.3.3.161

Harner, M. (1990). The way of the shaman. New York, NY: Harper \& Row.

Hoffman, S., Gafni, S., \& Laub, B. (Eds.). (1994). Cotherapy with individuals, families, and groups. Northvale, NJ: Jason Aronson.

Hoffman, S., \& Laub, B. (2006). Innovative interventions in psychotherapy. Boca Raton, FL: Universal.

Jung, C. G. (1963). Memories, dreams, reflections. London, England: Random House.

Kabat-Zinn, J. (1990). Full catastrophe living: Using the wisdom of your body and mind to face stress, pain and illness. New York, NY: Dell.

Klein, G. (1976). Psychoanalytic theory. New York, NY: International Universities Press.

Koestler, A. (1978). Janus: A summing up. New York, NY: Random House.

Laub, B. (2001). The healing power of resource connection in the EMDR protocol. The EMDRIA Newsletter, 21-27.

Laub, B., \& Weiner, N. (2007). The pyramid model Dialectical polarity in therapy. Journal of Transpersonal Psychology, 39(2), 199-221.

Laub, B., \& Weiner, N. (2011). A developmental/integrative perspective of the Recent Traumatic Episode Protocol (R-TEP). Journal of EMDR Practice and Research, 5(2), 57-72. doi:10.1891/1933-3196.5.2.57

Laub, B., \& Weiner, N. (2013). A dialectical perspective of trauma processing. International Journal of Integrative Psychotherapy, 4(2), 24-39.

Lee, C. W., Taylor, G., \& Drummond, P. D. (2006). The active ingredient in EMDR: Is it traditional exposure or dual focus of attention? Clinical Psychology \& Psychotherapy, 13(2), 97-107. doi:10.1002/ cpp.479

Levine, P. A., \& Frederick, A. (1997). Waking the tiger: Healing trauma. Berkeley, CA: North Atlantic Books.

Lynch, T. R., Chapman, A. L., Rosenthal, M. Z., Kuo, J. R., \& Linehan, M. M. (2006). Mechanisms of change in dialectical behavior therapy: Theoretical and empirical observations. Journal of Clinical Psychology, 62(4), 459-480. doi:10.1002/jclp.20243

Maxfield, L., Melnyk, W. T., \& Hayman, C. A. G. (2008). A working memory explanation for the effects of eye movements in EMDR. Journal of EMDR Practice and Research, 2(4), 247-261. doi:10.1891/1933-3196.2.4.247

Perls, F. S. (1959). Gestalt therapy verbatim. New York, NY: Real People.
Pipp, S. (1990). Sensorimotor representational internal working models of self, other, and relationship: Mechanisms of connection and separation. In D. Cicchetti \& M. Beeghly (Eds.), The self in transition: Infancy to childhood (pp. 243-264). Chicago, IL: The University of Chicago Press.

Shapiro, E., \& Laub, B. (2008). Early EMDR intervention (EEI): A summary, a theoretical model, and the Recent Traumatic Episode Protocol (R-TEP). Journal of EMDR Practice and Research, 2(2), 79-96. doi:10.1891/19333196.2.2.79

Shapiro, E., \& Laub, B. (2009). The new Recent Traumatic Episode Protocol (R-TEP). In M. Luber (Ed.), Eye Movement Desensitization and Reprocessing (EMDR) scripted protocols: Basics and special situations (pp. 251-270). New York, NY: Springer Publishing.

Shapiro, E., \& Laub, B. (2013). The Recent Traumatic Episode Protocol (R-TEP): An integrative protocol for Early EMDR Intervention (EEI). In M. Luber (Ed.), Implementing EMDR early mental health interventions for manmade and natural disasters: Models, scripted protocols, and summary sheets (pp. 193-207). New York, NY: Springer Publishing.

Shapiro, F. (1995). Eye movement desensitization and reprocessing: Basic principles, protocols and procedures. New York, NY: Guilford Press.

Shapiro, F. (2001). Eye movement desensitization and reprocessing: Basic principles, protocols and procedures (2nd ed.). New York, NY: Guilford Press.

Shapiro, F. (2007). EMDR, adaptive information processing, and case conceptualization. Journal of EMDR Practice and Research, 1(2), 68-87. doi:10.1891/1933-3196.1.2.68

Siegel, D. J. (1999). The developing mind. New York, NY: Guilford Press.

Siegel, D. J. (2007). The mindful brain: Reflection and attunement in the cultivation of well-being. New York, NY: Norton.

Siegel, D. J. (2010). The mindful therapist: A clinician's guide to mindsight and neural integration. New York, NY: Norton.

Smuts, J. C. (1926). Holism and evolution. London, England: MacMillan.

Solomon, R. M., \& Shapiro, F. (2008). EMDR and the adaptive information processing model. Journal of EMDR Practice and Research, 2, 315-325. doi:10.1891/19333196.2.4.315

Stone, H., \& Winkelman, S. (1985). Embracing ourselves. Voice dialogue manual. Marina del Rey, CA: DeVorss \& Company.

van der Hart, O., van Dijke, A., van Son, M., \& Steel, K. (2000). Somatoform dissociation in traumatized World War I combat soldiers: A neglected clinical heritage. Journal of Trauma and Dissociation, 1(4), 33-66. doi:10.1300/J229v01n04_03

Watts, A. W. (1963). The two hands of God: The myth of polarity. New York, NY: Collier Books. 
Wilber, K. (1996). A brief history of everything. Boston, MA: Shambhala.

Williams, H. L. (1989). Hegel, Heraclitus and Marx's dialectic. Hemel Hempstead, England: Harvester Wheatsheaf.
Merci d'adresser toute correspondance concernant cet article à Brurit Laub, 12 Hadar St., Rehovot 7646612, Israël. E-mail: bruritlaub7@gmail.com 